\title{
A closer looking at dimming and brightening in China during 1961-2005
}

\author{
X. Xia \\ LAGEO, Institute of Atmospheric Physics, Chinese Academy of Sciences, Beijing, 100029, China \\ Received: 2 March 2010 - Revised: 22 April 2010 - Accepted: 7 May 2010 - Published: 17 May 2010
}

\begin{abstract}
This study investigates dimming and brightening of surface solar radiation (SSR) during 1961-2005 in China as well as its relationships to total cloud cover (TCC). This is inferred from daily ground-based observational records at 45 pyranometer stations. A statistical method is introduced to study contributions of changes in the frequency of TCC categories and their atmospheric transparency to the secular SSR trend. The surface records suggest a renewed dimming beyond 2000 in North China after the stabilization in the 1990s; however, a slight brightening appears beyond 2000 in South China. Inter-annual variability of SSR is negatively correlated with that of TCC, but there is a positive correlation between decadal variability of SSR and TCC in most cases. The dimming during 1961-1990 is exclusively attributable to decreased atmospheric transparency, a portion of which is offset by TCC frequency changes in Northeast and Southwest China. The dimming during 1961-1990 in Northwest and Southeast China primarily results from decreased atmospheric transparency under all sky conditions and the percentage of dimming stemming from TCC frequency changes is $11 \%$ in Northwest and 2\% in Southeast China. Decreased atmospheric transparencies during 19912005 in North China in most cases lead to the dimming. TCC frequency changes also contribute to the dimming during this period in North China. This feature is more pronounced in summer and winter when TCC frequency changes can account for more than $80 \%$ of dimming. In South China, increased atmospheric transparencies lead to the brightening during 1991-2005. A substantial contribution by TCC frequency changes to the brightening is also evident in spring and autumn.
\end{abstract}

Keywords. Atmospheric composition and structure (Transmission and scattering of radiation)

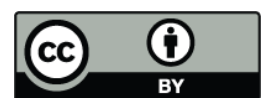

Correspondence to: X. Xia

(xxa@mail.iap.ac.cn)

\section{Introduction}

Based on analysis of inter-annual changes in solar radiation at the Earth's surface (SSR) from pyranometer measurements or proxy data, a number of studies have pointed to a widespread decrease of SSR from the early 1960s to the late 1980s ("global dimming") (Liepert, 2002; Stanhill and Cohen, 2001). More recent studies have used data records updated to include the 1990s, and these studies suggest that the decline in SSR diminished during the 1980s, and leveled off or even reversed towards an increase during the 1990s ("brightening") (Wild et al., 2009a, b, and references therein). A few studies have shown a significant decline in SSR during 1961-1990 over much of China, especially in eastern China where SSR has declined by more than $6 \%$ per decade (Li and Zhou, 1998; Che et al., 2005; Liang and Xia, 2005; Ren et al., 2005; Shi et al., 2008; Xia et al., 2006). In the 1990s, the downward trend leveled off or even changed sign (Che et al., 2005; Xia et al., 2006). Analysis of SSR data updated through 2005 from 9 pyranometer sites in China suggests that to some extent the phase of stabilization/brightening during the 1990s may no longer be continuing in the new millennium (Wild, 2009b). This is supported by analysis of sunshine duration data updated through 2005 from 618 sites in China (Xia, 2010). The decrease in SSR between the 1960s and 1980s is accompanied by concurrent declines in total cloud cover (TCC) and rainy days (Kaiser, 1998; Liang and Xia, 2005). An analysis using the more reliably observed frequencies of cloud-free and overcast sky shows that cloud-free days have increased $0.60 \%$ and overcast days have decreased $0.78 \%$ per decade in China during 1954-2001 (Qian et al., 2006). Concurrent declines in TCC and SSR before the 1990s lead to the speculation that there should be other causes for the dimming, among which aerosols emitted as a result of human activities is the most likely cause. Aerosol optical depth is estimated to increase from 0.38 in 1960 to 0.47 in 1990 (Luo et al., 2001; Qiu and

Published by Copernicus Publications on behalf of the European Geosciences Union. 
Yang, 2000). Visibility was reduced by $35 \%$ from the 1960 s to the 1980s in south China and frequencies of good visibility $(>20 \mathrm{~km}$ ) decreased by more than $20 \%$ per decade in eastern China (Che et al., 2007; Liang and Xia, 2005). Some sunlight would be prevented from reaching Earth's surface as a result of increased aerosol loading. Using synoptic cloud and satellite retrievals of SSR under clear and cloudy skies over China, it was pointed out that aerosols are major modulators of SSR during 1971-1989 and half of the 1990-2002 SSR trend can be attributed to a reduction in cloud cover (Norris and Wild, 2009). It should be noted that the cause of the decadal variability of SSR is still not fully understood and certainly it needs further study.

Using daily SSR and TCC data from 45 sites in China during 1961-2005, the first objective of this study is to investigate how SSR is further evolving into the new millennium. Secondly, correlations between SSR and TCC have been studied to determine the degree of agreement between the high and low frequency parts of both quantities. Thirdly, a statistical method is applied to determine how much of any SSR change is attributable to changes in the frequency of TCC categories and how much to changes in their atmospheric transparencies.

\section{Data and methodology}

There are 122 pyranometer sites in China. But only 45 sites have no missing data of no more than 2 years during 19612005 , and these sites are used in the analysis. The data are provided by the Climate Data Center, Chinese Meteorological Administration (CDC/CMA). These data have been used to detect secular trends in SSR and cloud cover (Kaiser, 1998; Qian et al., 2007; Shi et al., 2008; Xia et al., 2006). The Yanishevsky thermoelectric pyranometer was used to measure global SSR before about 1990 and since then the DFY-4 pyranometer was used. The error has been estimated to be less than 5\% (Ma et al., 1998; Qiu et al., 2008; Shi et al., 2008). Ground-based observations of TCC (in units of tenths of sky cover) are based on subjective estimates by experienced individuals at stations. Observations are carried out according to the recommendations of the WMO (World Meteorological Organization). Quality assurance checks, including gross errors and the consistency of calendar dates, were performed by CDC/CMA (Ma et al., 1998).

24-30 years worth of data are required to obtain mean value of either cloud cover or SSR being within $1 \%$ accuracy with 95\% confidence (Hoyt, 1978). These lengths of time are comparable to the periods of time on which climatic changes occur. This implies that one site alone is not adequate to determine climatic trends in cloud cover or in SSR. Rather, a network of stations of sufficient density to determine mean cloud cover or SSR is needed for climatic change studies. Therefore, the 45 sites are classified into four subclasses and the regional averages of SSR and TCC are ana- lyzed. Figure 1 presents the four sub-regions: (1) Northeast (NE); (2) Northwest (NW); (3) Southeast (SE); (4) Southwest (SW).

All data were converted to departures from normal (anomalies), with normals approximated by period means of TCC and SSR data. The monthly anomalies of TCC and SSR have been averaged by season (winter is defined as December, January, and February, spring as March, April, and May, summer as June, July, and August, and autumn as September, October, and November) and an annual average has been obtained from the average of the four seasons. Annual and seasonal deviations from the long-term mean have been determined for each station, and these deviations have been averaged for stations within each of the four regions. A simple and robust estimator of trends per decade is computed based on the non-parametric Kendall rank correlation (tau), which is used to assess the temporal development of SSR and TCC.

\section{Spatio-temporal variability of SSR and TCC, as well as their correlations}

The annual and seasonal time series of SSR and TCC, together with their fits from the robust locally weighted "Lowess" regression algorithm for a better visualization of long-term and decadal variability, are presented in this section. In order to determine the degree of agreement between SSR and TCC, the correlation coefficient between the time series of SSR and TCC is calculated and its significance is determined using a two-tail $t$ test. In the first step of correlation calculations, the raw (i.e., non-detrended) time series of SSR and TCC anomalies are used. In order to distinguish between the decadal and inter-annual agreement of the two measures, we detrended the raw time series and recalculated the correlations between the higher (inter-annual variability) and the lower (multiannual to decadal variability) frequency parts of each series. A fit is determined by the robust locally weighted regression algorithm "Lowess" to represent the long-term signal, which is subtracted from the raw series to derive the detrended residuals or high-frequency changes (Makowski et al., 2009). Given that SSR generally turned from dimming to brightening and the DFY-4 pyranometer replaced the Yanishevsky thermoelectric pyranometer at the beginning of 1990s, therefore, analyses are applied to data for the years of 1961-1990 and for the years of 1991-2005 separately.

\subsection{NW region}

The linear trends per decade during 1961-1990 and during 1991-2005 are $-6.4 \mathrm{~W} \mathrm{~m}^{-2}$ and $-2.9 \mathrm{~W} \mathrm{~m}^{-2}$, indicating a renewal of dimming during the new century (Fig. 2a). The seasonal time series of SSR resembles the annual time series except that the spring SSR series levels off beyond 2000. A weak upward tendency is observed for the annual series 


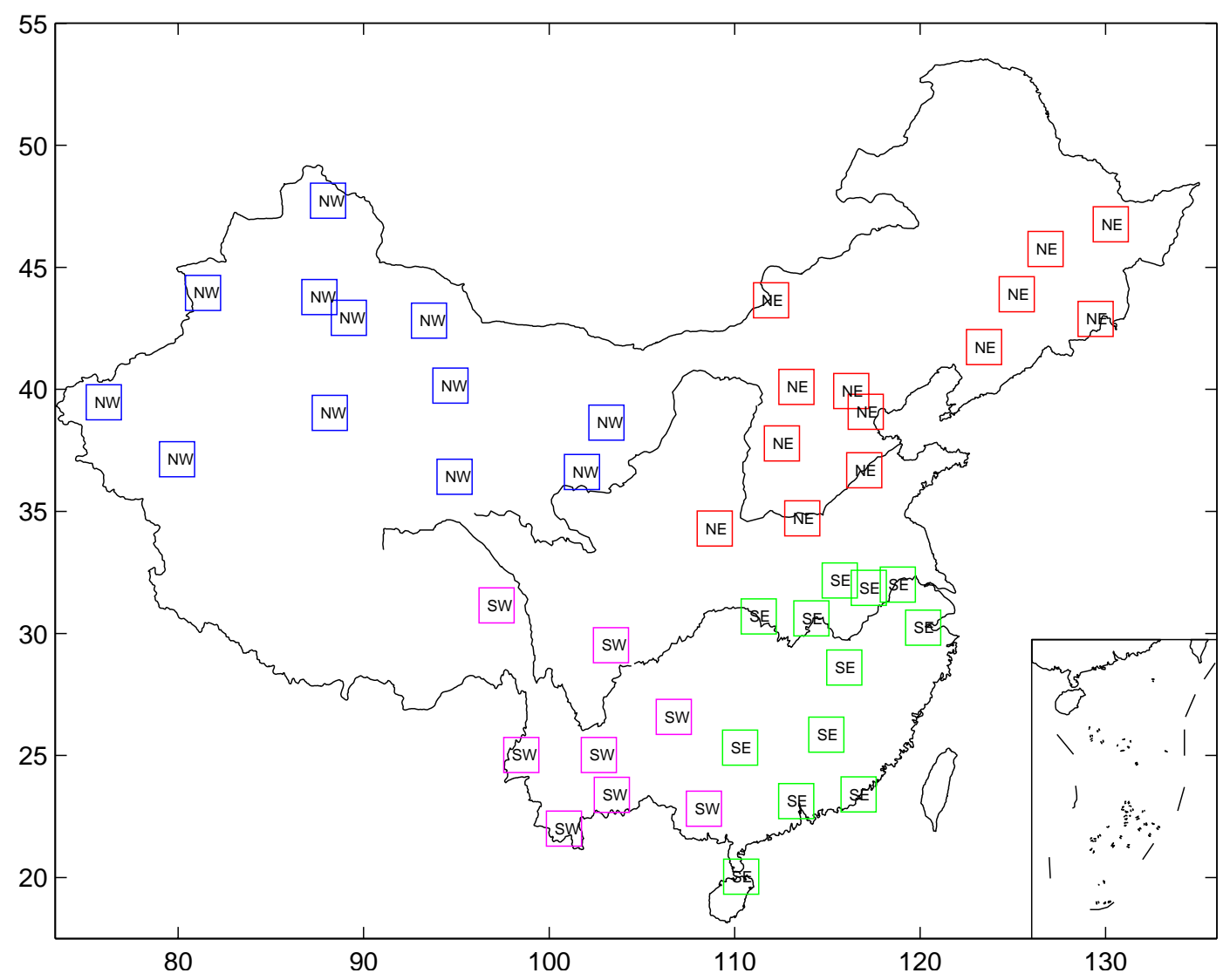

Fig. 1. Locations of pyranometer stations in China in are classified into four sub-regions, i.e., Northwest (NW), Northeast (NE), Southeast (SE) and Southwest (SW).

of TCC. The seasonal TCC series during 1961-1990 (except for the autumn series) also show an increasing trend ( $\sim 0.1$ tenths). A strong increase in TCC is observed during 1991-2005 in all seasons except spring. The largest increase in TCC (0.49 tenths) during 1991-2005 appears in autumn, which is in good agreement with the most pronounced decreasing trend in SSR $\left(-6.5 \mathrm{~W} \mathrm{~m}^{-2}\right)$. Notice also in Fig. 2 that inter-annual variability of SSR follows that of TCC and there is a strong and significant inverse correlation between them at seasonal to annual resolutions (see correlation coefficients in Table 2). The low frequency component of SSR is also inversely correlated to the corresponding component of TCC in nearly all cases except for the autumn series during 1961-1990. Note in Fig. 2 that during 1991-2005, a decreasing trend in SSR is closely related to an increasing tendency of TCC. This indicates that a portion of a renewed dimming in the new millennium is likely attributable to TCC changes.

\subsection{NE region}

The annual SSR decreases significantly by $7.0 \mathrm{~W} \mathrm{~m}^{-2}$ per decade during 1961-1990. There is some indication for a renewal of dimming beyond 2000 after the stabilization in the 1990s (Fig. 3) and SSR decreases by $3.9 \mathrm{~W} \mathrm{~m}^{-2}$. The seasonal time series of SSR also decreases significantly from 1960s to 1980s (see trends in Table 1). Notice in Fig. 3b-e that, for the seasonal series, a renewed dimming trend appears beyond 2000. The annual TCC series also show a decreasing trend from the 1960 s to the end of 1990s, and afterwards there is an increasing trend that is mostly because TCC increases in summer and winter (Fig. 3). The interannual variability of SSR is strongly inversely related to that of TCC and the correlation coefficients exceed -0.75 . One interesting feature is that the low frequency components of SSR and TCC show a decreasing trend from the 1960s to the 1980 s, thereby leading to a significant positive correlation (Table 2). This implies that the dimming during this period cannot be explained by the decadal variability of TCC. On the contrary, the decadal variability of SSR inversely follows that of TCC since the 1990s and the negative correlation coefficients exceed -0.90 in all seasons except in autumn. This suggests that the dimming during 1991-2005 can be partly attributable to the decadal variability of TCC. 

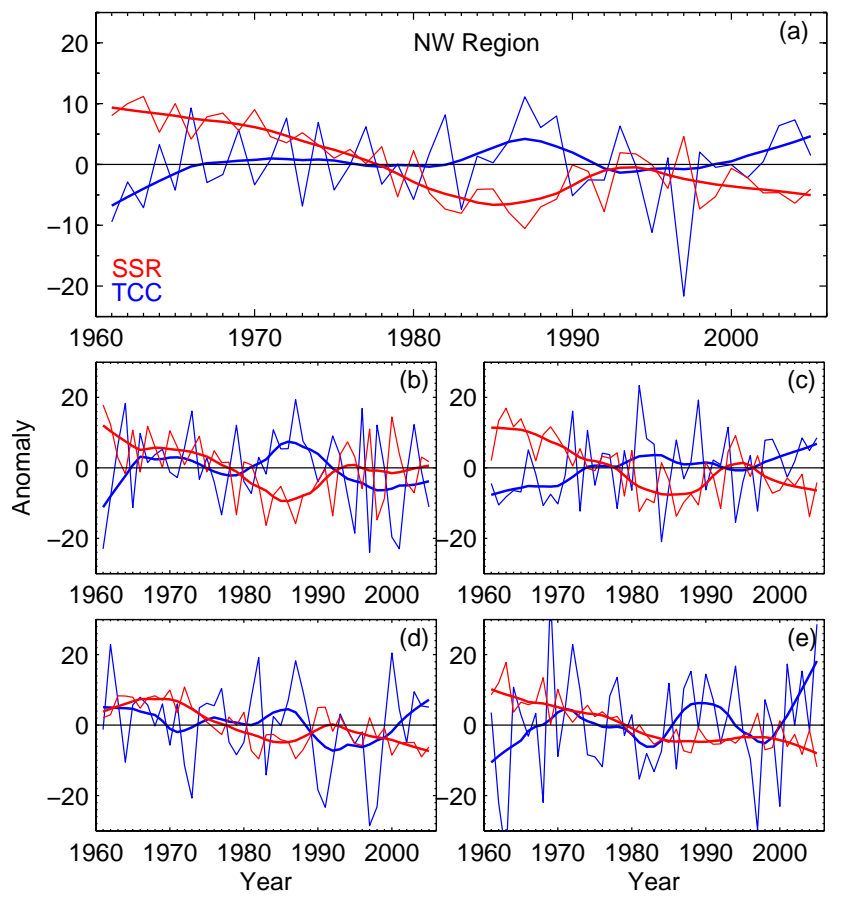

Table 1. The annual and seasonal trends in surface solar radiation (SSR) and total cloud cover (TCC) during 1961-1990 and during 1991-2005.

\begin{tabular}{|c|c|c|c|c|c|}
\hline \multirow{2}{*}{ Region } & \multirow{2}{*}{ Trend } & \multicolumn{2}{|c|}{ 1961-1990 } & \multicolumn{2}{|c|}{ 1991-2005 } \\
\hline & & SSR & TCC & SSR & TCC \\
\hline \multirow{5}{*}{ NW } & ANN & -6.41 & 0.07 & -2.86 & 0.16 \\
\hline & MAM & -7.29 & 0.08 & 1.39 & -0.16 \\
\hline & JJA & -7.63 & 0.12 & -5.28 & 0.17 \\
\hline & SON & -4.82 & -0.03 & -6.48 & 0.49 \\
\hline & DJF & -5.93 & 0.10 & -1.67 & 0.26 \\
\hline \multirow{5}{*}{$\mathrm{NE}$} & ANN & -7.02 & -0.12 & -3.94 & 0.23 \\
\hline & MAM & -7.51 & -0.17 & -1.37 & 0.04 \\
\hline & JJA & -9.76 & -0.08 & -7.88 & 0.33 \\
\hline & SON & -4.56 & -0.21 & -4.02 & 0.10 \\
\hline & DJF & -6.33 & -0.02 & -3.53 & 0.50 \\
\hline \multirow{5}{*}{ SE } & ANN & -12.00 & 0.02 & 3.68 & -0.02 \\
\hline & MAM & -12.22 & 0.06 & 12.72 & -0.41 \\
\hline & JJA & -15.19 & -0.03 & 1.34 & 0.04 \\
\hline & SON & -9.88 & -0.04 & -0.34 & 0.20 \\
\hline & DJF & -10.80 & 0.12 & 0.10 & 0.13 \\
\hline \multirow{5}{*}{ SW } & ANN & -2.83 & -0.09 & 3.64 & -0.01 \\
\hline & MAM & -4.37 & 0.03 & -1.56 & 0.27 \\
\hline & JJA & -0.85 & -0.15 & 5.86 & -0.04 \\
\hline & SON & -4.35 & -0.03 & 4.99 & -0.22 \\
\hline & DJF & -1.68 & -0.20 & 5.42 & -0.15 \\
\hline
\end{tabular}

Fig. 2. Time series plots of annual anomalies of surface solar radiation (SSR), total cloud cover (TCC), and low cloud cover (LCC), plotted together with the smoothed series using robust locally weighted regression algorithm "Lowess" in Northwest China: (a); spring (b); summer (c); autumn (d); winter (e).

\subsection{SE region}

The annual SSR series decreases by $12.0 \mathrm{~W} \mathrm{~m}^{-2}$ per decade during 1961-1990 (Fig. 4 and Table 1). Afterward, a positive trend $\left(3.7 \mathrm{~W} \mathrm{~m}^{-2}\right)$ appears up to the end of the analyzed period. The temporal behavior of seasonal SSR time series generally resembles that of the annual series. However, the turning point from dimming to brightening appears early (at the beginning of the 1980s) in summer. The brightening is not significant in autumn and winter during 1991-2005. An obvious upward tendency is observed for the annual TCC series from the 1960s to the beginning of 1970s and TCC decreases gradually thereafter. The spring TCC series resembles the annual series. However, for other seasons, TCC shows a very large decadal fluctuation and the seasonal TCC shows a weak increasing trend during 1991-2005. The short-term variability of SSR is negatively correlated to that of TCC (Table 2). There is a strong inverse correlation between the low frequency component of SSR and TCC for the spring time series during both periods. In Fig. 4, it is observed that an upward tendency of SSR during 1991-2005 is generally accompanied by a decreasing trend in TCC and thereby, the correlation coefficients between the low frequency components are generally negative. This suggests that the SSR variability during 1991-2005 is closely related to that of TCC.

\subsection{SW region}

The annual time series of SSR shows a decreasing trend from the 1960s to the end of 1990s. Dimming is strong for the spring and autumn time series. Brightening seems to appear beyond 2000, especially for the spring, summer, and autumn time series (Fig. 5b-d). The annual time series of TCC shows a significant decreasing trend during 1961-2000 and thereafter an increasing trend. The seasonal TCC anomalies during 1961-1990 generally show a decreasing trend but during 1991-2005, they show an increasing trend or level off.. We can also see a strong negative correlation between the high frequency portions of SSR and TCC in this region. For the low frequencies, an inverse correlation between SSR and TCC is often intervened by a positive correlation. The inverse correlation appears during 1991-2005 for the summer, autumn, and winter time series and the correlation coefficients are $-0.99,-0.99$, and -0.87 , respectively (see Table 2). 
Table 2. Correlation coefficients between annual and seasonal time series of surface solar radiation and total cloud cover. "Raw" denotes coefficients for the original anomalies time series; "High" denotes the remaining high-frequency, inter-annual variations after detrending with the Lowess algorithm; and "High" denotes the correlations of the low-frequency part of the original time series as derived from Lowess. Coefficients given in bold are significant at the $95 \%$ level.

\begin{tabular}{|c|c|c|c|c|c|c|c|c|c|c|c|}
\hline & \multicolumn{6}{|c|}{ 1961-1990 } & \multicolumn{5}{|c|}{$1991-2005$} \\
\hline & & Annual & Spring & Summer & Autumn & Winter & Annual & Spring & Summer & Autumn & Winter \\
\hline \multirow{3}{*}{ NW } & High & -0.59 & -0.68 & -0.71 & -0.73 & -0.83 & -0.49 & -0.81 & -0.34 & -0.56 & -0.88 \\
\hline & Low & -0.70 & -0.68 & -0.95 & 0.07 & -0.40 & -0.89 & -0.32 & -0.98 & -0.94 & -0.96 \\
\hline & Raw & -0.46 & -0.59 & -0.63 & -0.42 & -0.51 & -0.55 & -0.79 & -0.49 & -0.64 & -0.90 \\
\hline \multirow{3}{*}{$\mathrm{NE}$} & High & -0.76 & -0.86 & -0.75 & -0.75 & -0.87 & -0.82 & -0.89 & -0.89 & -0.87 & -0.83 \\
\hline & Low & 0.86 & 0.72 & 0.73 & 0.62 & 0.10 & $-\mathbf{1 . 0 0}$ & -0.94 & -0.98 & 0.51 & -0.98 \\
\hline & Raw & 0.19 & -0.21 & -0.09 & -0.03 & -0.47 & -0.87 & -0.90 & -0.91 & -0.82 & -0.90 \\
\hline \multirow{3}{*}{ SE } & High & $-\mathbf{0 . 8 3}$ & -0.77 & -0.82 & -0.90 & -0.97 & -0.75 & -0.84 & -0.72 & -0.93 & -0.96 \\
\hline & Low & -0.08 & -0.92 & -0.14 & 0.19 & -0.25 & -0.74 & -0.97 & -0.41 & -0.48 & -0.60 \\
\hline & Raw & -0.37 & -0.59 & -0.43 & -0.57 & -0.79 & -0.68 & -0.91 & -0.69 & -0.89 & -0.95 \\
\hline \multirow{3}{*}{ SW } & High & -0.71 & -0.70 & -0.88 & -0.67 & -0.87 & -0.36 & -0.34 & -0.67 & -0.68 & -0.85 \\
\hline & Low & 0.75 & -0.18 & -0.45 & -0.21 & 0.35 & 0.22 & 0.28 & -0.99 & -0.99 & -0.87 \\
\hline & Raw & -0.17 & -0.64 & -0.72 & -0.51 & -0.66 & -0.38 & -0.38 & -0.66 & -0.75 & -0.85 \\
\hline
\end{tabular}

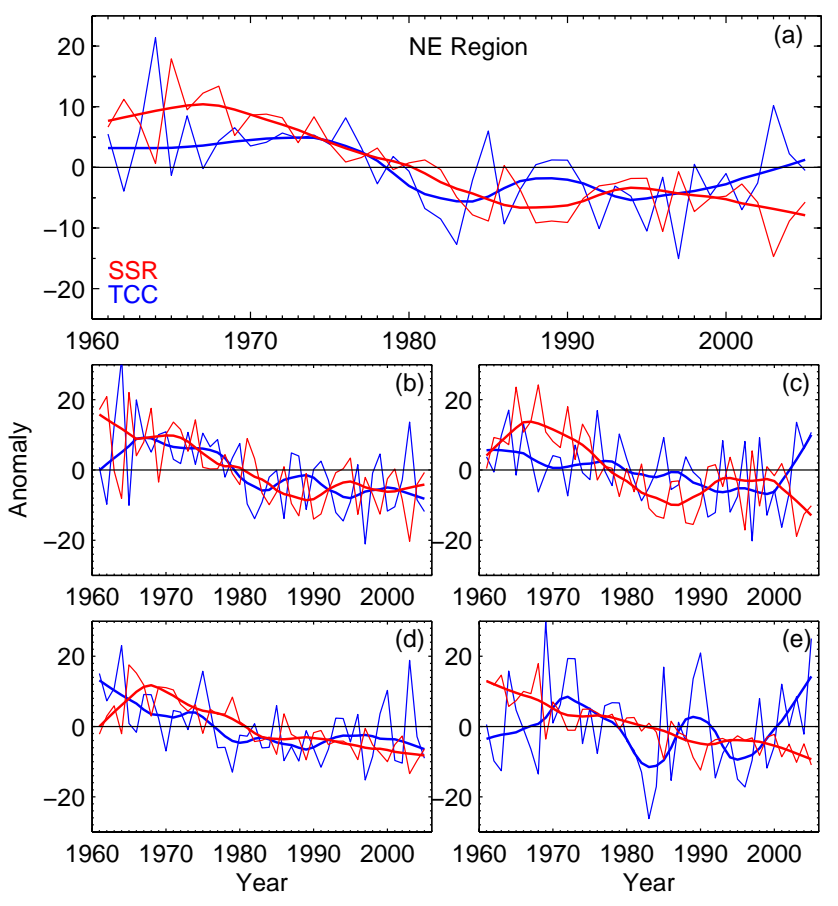

Fig. 3. The same as in Fig. 2, but in Northeast China.

\section{Contributions of changes in the frequency of cloud cover categories and their atmospheric transparency to the secular trend in SSR}

Notice in Sect. 3 that there is a very complex spatio-temporal variability of SSR. We can get some hints about the role that
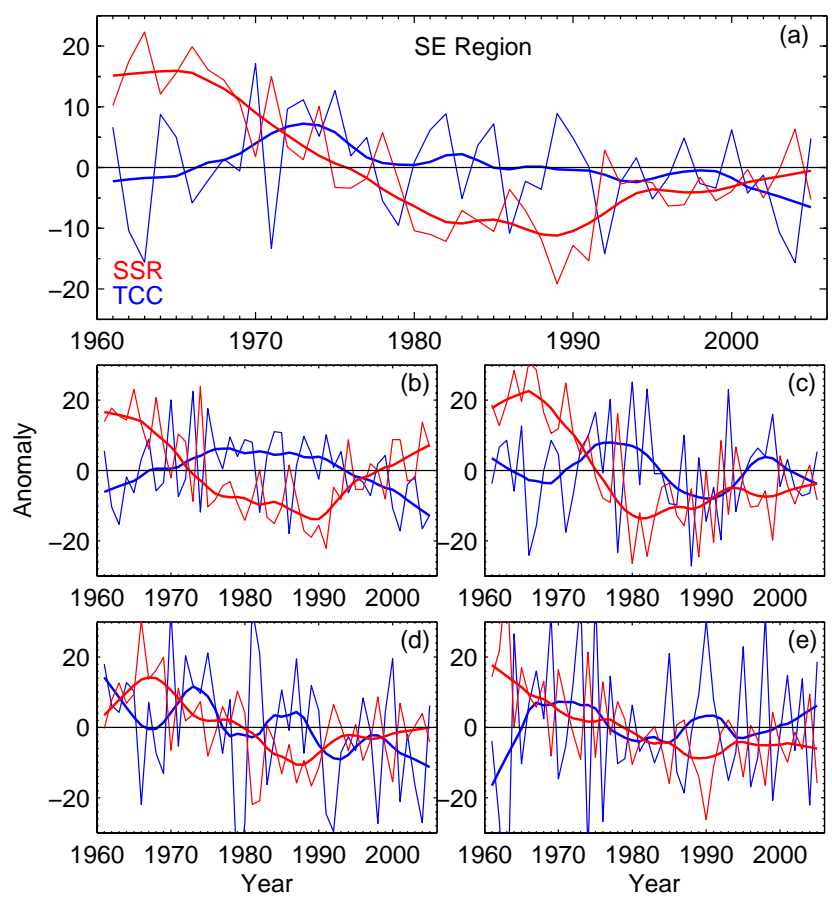

Fig. 4. The same as in Fig. 2, but in Southeast China.

TCC plays in the interannual and decadal variability of SSR through determining the degree of agreement between the high and low frequency parts of SSR and TCC. However, further study is required to understand how much of any SSR change is attributable to a change in the frequency of TCC categories and how much to changes in the atmospheric 


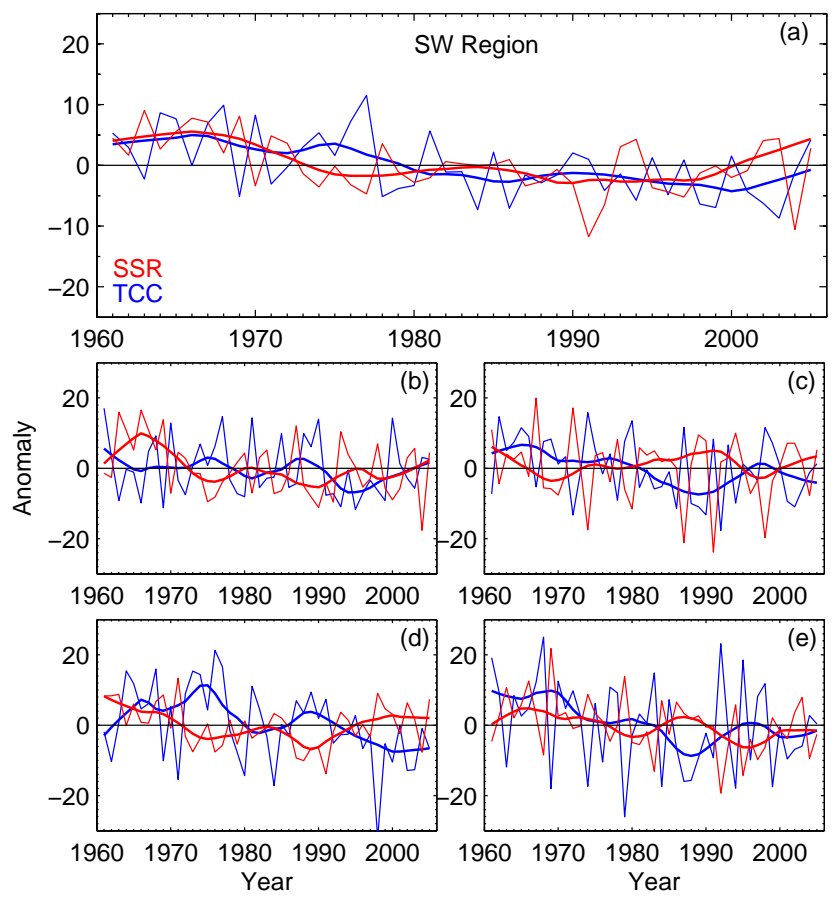

Fig. 5. The same as in Fig. 2, but in Southwest China.

transparency. In order to quantitatively estimate the contribution of TCC variability to that of SSR, eleven TCC categories are defined, i.e., TCC $=0,0-1,1-2,2-3,3-4,4-5,5-$ $6,6-7,7-8,8-9,9-10$ tenths, representing conditions from clear sky, cloudy sky, to overcast. Following the method introduced by Karl and Knight (Karl and Knight, 1998) to study contributions of precipitation frequency and intensity to secular trends of precipitation amount, the proportion of any trend in total SSR that is attributable to changes in frequency of any TCC category versus changes in atmospheric transparency is studied. This is calculated for the frequency component by determining the average SSR $\left(E_{\mathrm{g}}^{-}\right)$for any TCC category and the trend in the frequency of TCC categories $\left(\mathrm{TRD}_{\mathrm{f}}\right)$. Then the change in SSR due to the trend in the frequency of TCC categories is simply defined by

$\mathrm{TRD}_{\mathrm{CC}}=E_{\mathrm{g}}^{-}\left(\mathrm{TRD}_{\mathrm{f}}\right)$

In this analysis, $\mathrm{TRD}_{\mathrm{CC}}$ is expressed as a percentage of the mean seasonal or annual total SSR. For the atmospheric transparency component, the trend is directly calculated as a residual using the expression

$\mathrm{TRD}_{\mathrm{AT}}=\mathrm{TRD}-\mathrm{TRD}_{\mathrm{CC}}$

where TRD is the trend in total SSR for specific TCC class intervals. The analysis is applied to data during 1961-1990 and during 1991-2005 separately for the same reason.

\subsection{NW region}

As shown in Fig. 6 (upper panel), an outstanding feature during 1961-1990 is that atmospheric transparency for all TCC categories decreases by about $0.25 \%$ per decade. This is the primary cause for the dimming. TCC frequency changes do support a portion of the dimming in all seasons except in autumn, and the percentages attributable to this are $16 \%$, $24 \%$, and $11 \%$ in spring, summer, and winter, respectively. TCC frequency change during 1991-2005 is the dominant factor determining SSR decadal variability (bottom panel of Fig. 6), although atmospheric transparency change still plays an important role. The percentages of the total SSR trend attributable to TCC frequency changes are $100 \%, 79 \%, 48 \%$, and $88 \%$ for 4 seasonal series.

\subsection{NE region}

The dimming during 1961-1990 (the upper panel of Fig. 7) is entirely attributable to a decline in SSR under all TCC categories. Decreased atmospheric transparency under any TCC category leads to total seasonal SSR declines of $4.7 \sim 5.9 \%$ per decade in four seasons. The overall effect of TCC frequency changes on SSR leads to a total seasonal SSR increase, thereby offsetting part of dimming that stems from decreased atmospheric transparency. Atmospheric transparency for most TCC categories during 1991-2005 still shows a decreasing trend (bottom panel of Fig. 7), which is an important cause for the dimming. A quite different feature during this period, as compared to the years 1961-1990, is that TCC frequency changes contribute to the dimming in all seasons except in spring. This feature is more pronounced in summer and winter and about $90 \%$ of dimming is explained by TCC frequency changes.

\subsection{SE region}

Dimming during 1961-1990 is primarily and perhaps even exclusively attributable to decreased atmospheric transparency (Fig. 8). A very interesting feature is that contributions by atmospheric transparency changes increase with TCC, and this feature is more pronounced in spring and summer. An outstanding feature during 1991-2005 is that nearly all atmospheric transparencies show an increasing trend, which results in the brightening of SSR. The brightening in spring is enhanced by changes in frequency of TCC categories that contributes to about $63 \%$ of the brightening. Increased atmospheric transparency is the unique reason for the brightening in summer and winter, about $26 \%$ and $57 \%$ of which is offset by TCC frequency changes. A significant decrease in the frequency of days with TCC less than 1 tenth in autumn leads to a decreasing trend in SSR, which is the exclusive cause for the dimming in this season. 

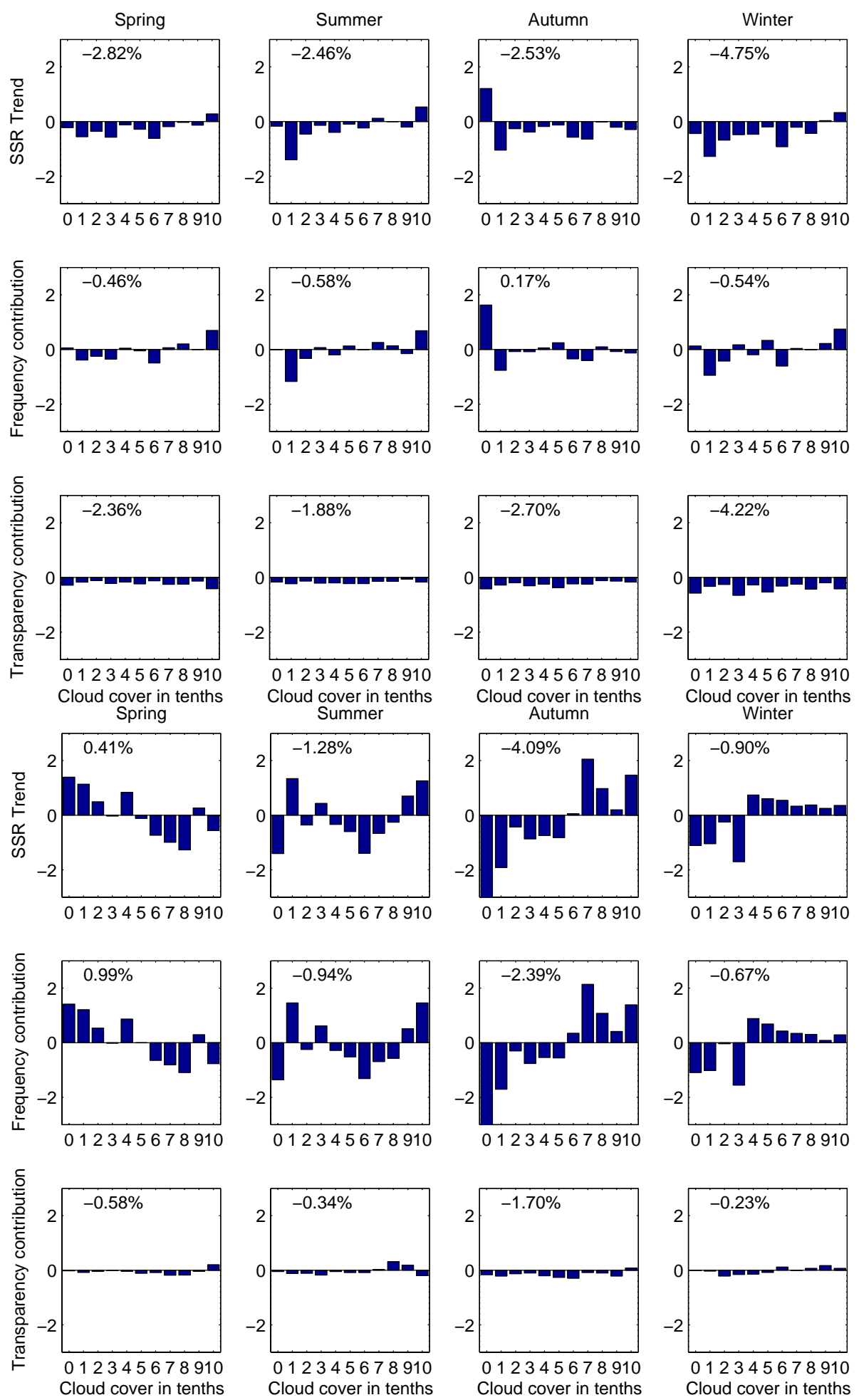

Fig. 6. Seasonal trends in Northwest China during 1961-1990 (upper panel) and during 1991-2005 (bottom panel) expressed as percent of mean SSR per decade for various categories of total cloud cover in tenths (upper); the contribution to the SSR trends attributed to trends in total cloud cover frequency (middle) and the contribution to the SSR trends attributed to trends in the atmospheric transparency (bottom). The values represent the sum of trends in SSR and the total contributions to those trends attributable to trends in total cloud cover frequency and trends in the atmospheric transparency. 

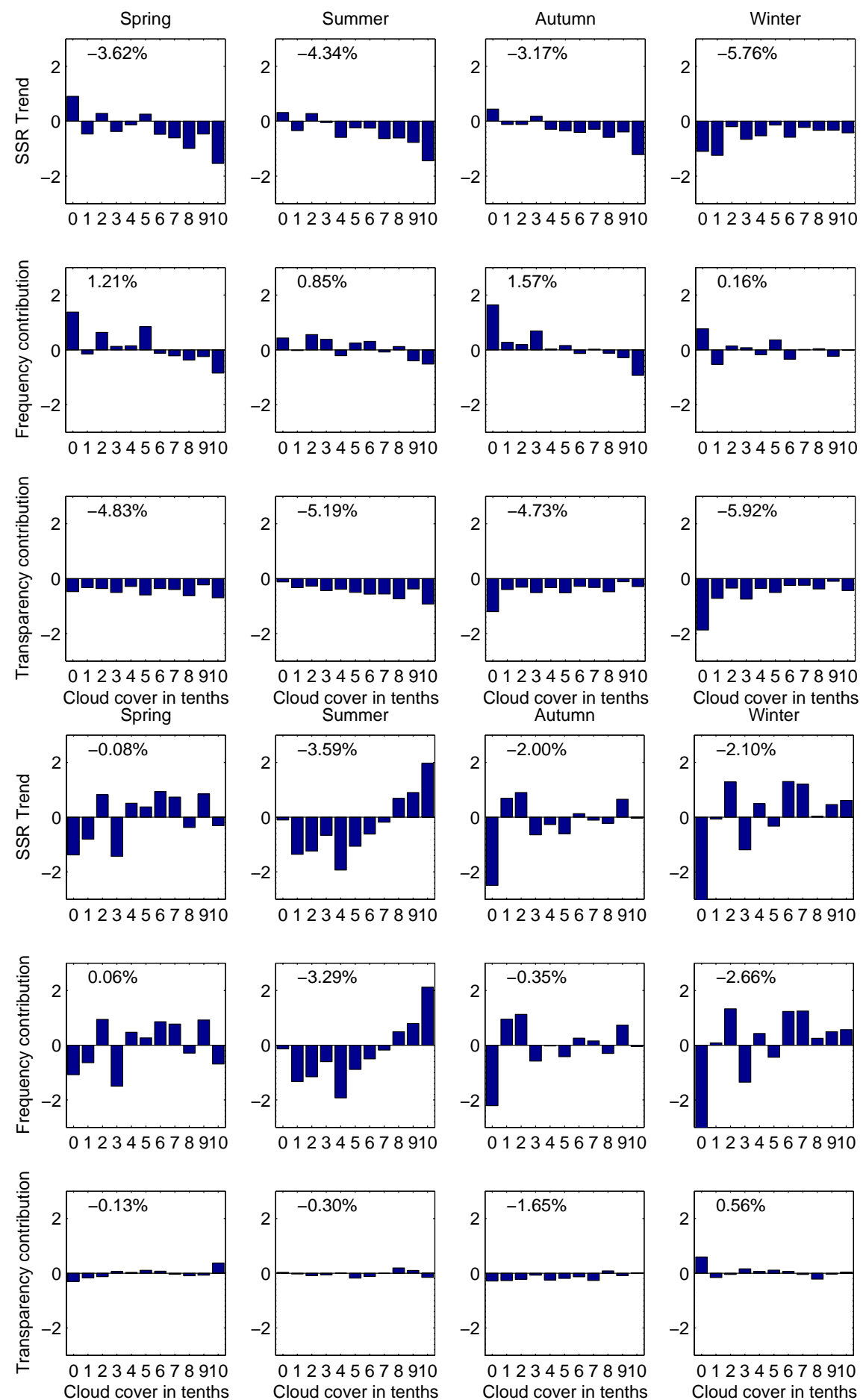

Fig. 7. The same as in Fig. 6, but in Northeast China.

\subsection{SW region}

Atmospheric transparency for any TCC category shows a decreasing trend during 1961-1990, which is the unique cause for the dimming (Fig. 9). The dimming is, to some extend, offset by a positive contribution to SSR due to TCC fre-

quency changes. This is more pronounced in summer and winter and the percentage of dimming offset by TCC frequency changes is $87 \%$ and $84 \%$, respectively. A renewed dimming in spring during 1991-2005 is entirely attributable to changes in frequencies of TCC categories. Changes in frequency of TCC categories contribute to a brightening by 

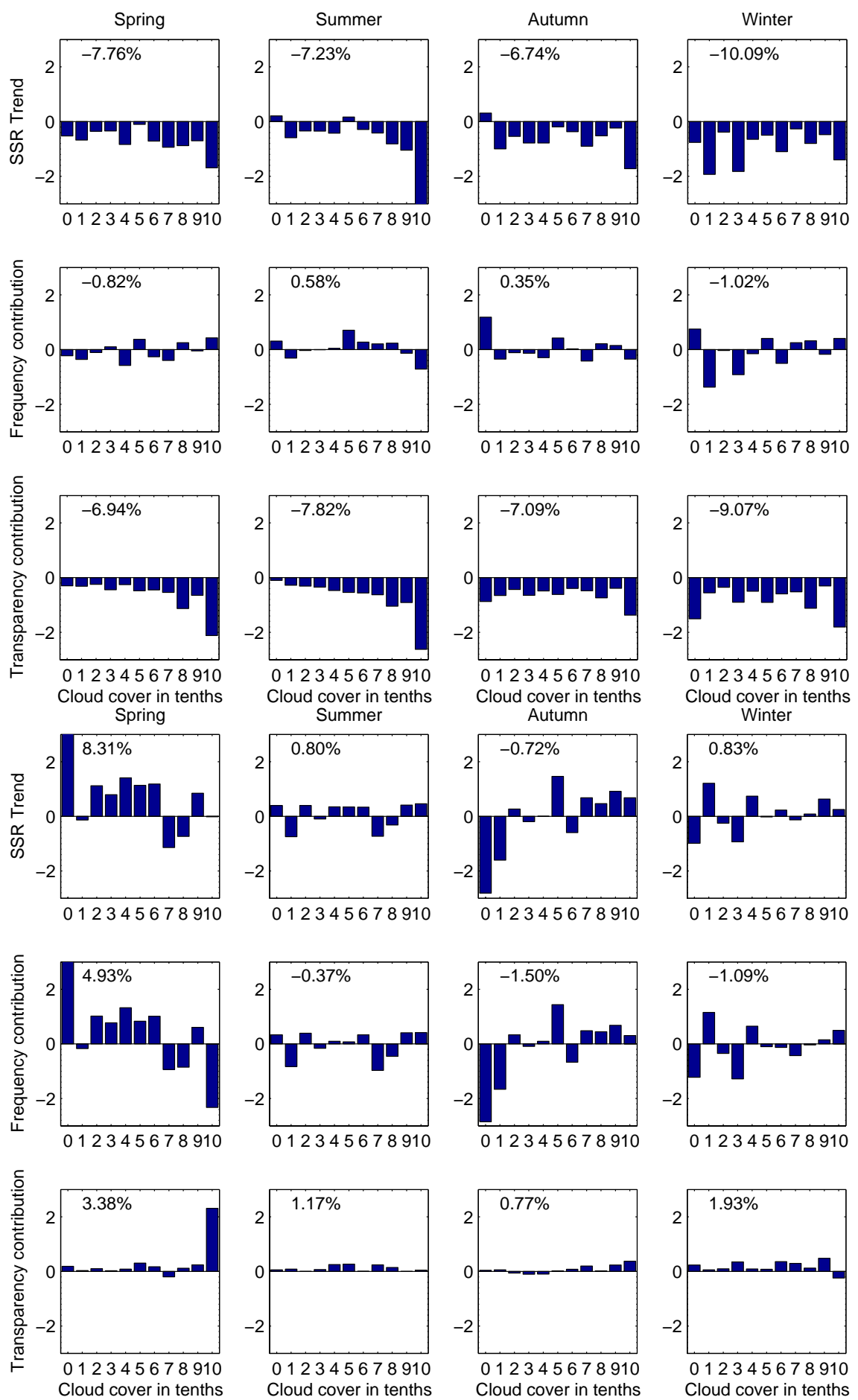

Fig. 8. The same as in Fig. 7, but in Southeast China.

$8 \%$ in summer and $53 \%$ in autumn during 1991-2005. The brightening in winter during 1991-2005 is completely attributable to increased atmospheric transparency (Fig. 9).

\section{Discussion}

TCC appears to be an important modulator to SSR, as revealed by a significantly negative correlation between the high frequency components of SSR and TCC. Correlation between the long-term behavior of SSR and TCC is 

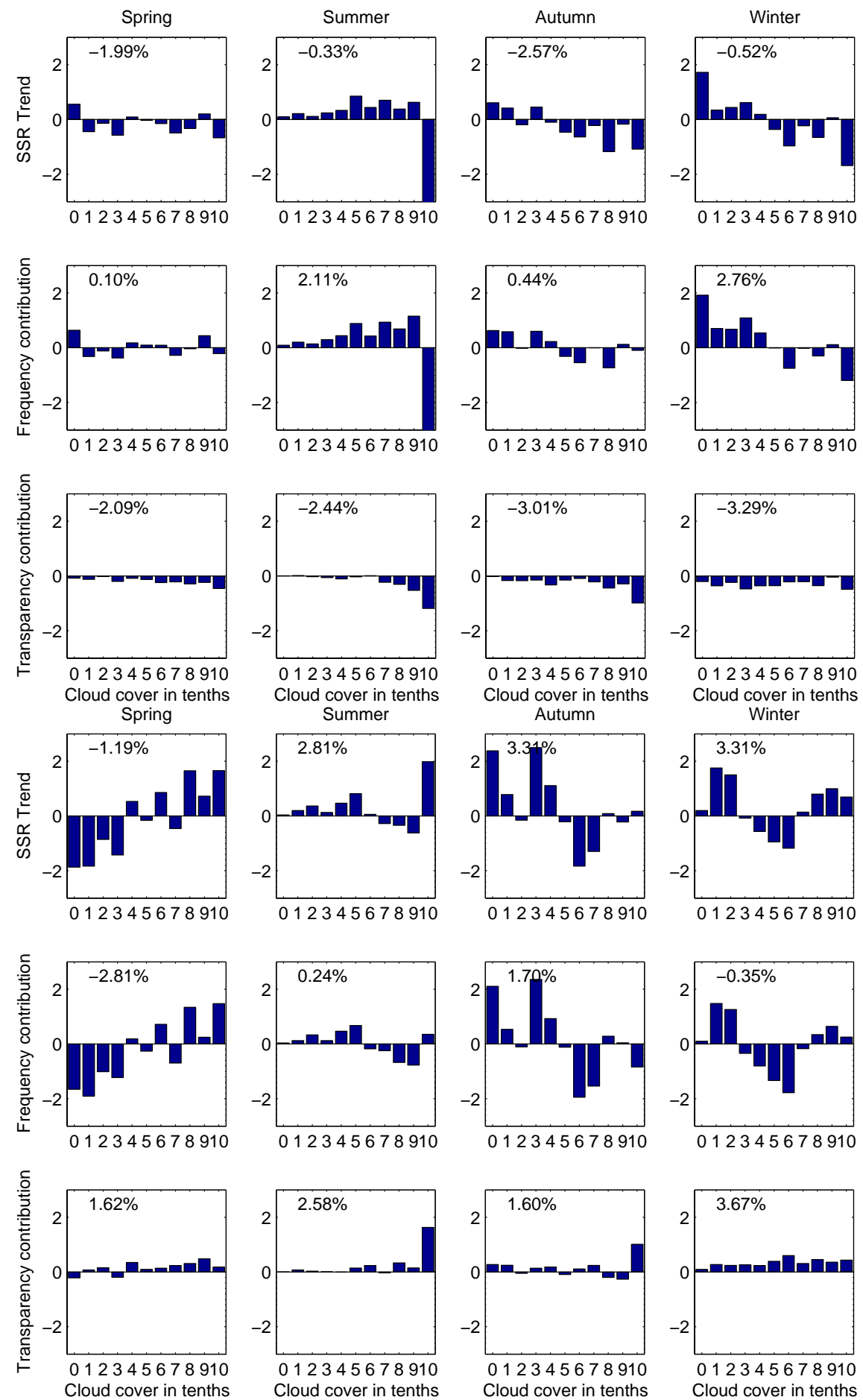

Fig. 9. The same as in Fig. 8, but in Southwest China.

somewhat complex. The dimming during $1961-1990$ is accompanied by a decreasing trend in TCC (Li et al., 1998; Che et al., 2005; Liang and Xia, 2005) and an increasing trend in clear days (Qian et al., 2006), indicating negligible contribution by TCC to the SSR trend over China (Norris and Wild,
2009). However, we can still see that a portion of the dimming is likely attributable to changes in TCC in a few cases. For example, TCC changes account to some extent for the dimming in northwest China in nearly all seasons except in autumn and in southeast China (in spring and winter). This is 
confirmed by negative correlations between the decadal scale behavior of SSR and TCC. Furthermore, this is reasserted by quantitative estimates of the contribution of TCC frequency changes to the total SSR trend. During 1991-2005, part of decadal SSR variability in most cases can be attributed to changes in TCC. This is in accordance with results of Norris and Wild (2009), but our results show spatial and seasonal variations of TCC contribution. There are many cases in which more than half of decadal SSR variability can be attributable to TCC changes. For example, more than half of dimming in northwest China (all seasons) and in northeast China (summer and winter) could be explained by TCC changes. However, we also observed that TCC made negligible contribution to the winter SSR trend in south China.

During 1961-1990, atmospheric transparency decreases for nearly all TCC categories. A decline in clear sky transparency is very possible a result of increased aerosol loading (Luo et al., 2001; Qiu and Yang, 2000). It was observed that the aerosol radiative effect on SSR under clear sky in North China Plain and the Yangtze Delta region is comparable to that of cloud effects (Li et al., 2007a, b; Xia et al., 2007). Note that the contribution by changes in atmospheric transparency to explained variance in SSR under cloudy and overcast conditions is generally comparable to that under clear sky. In south China, we can see that the contribution by changes in atmospheric transparency increases as TCC goes up in summer, which is likely associated with aerosol indirect effect.

\section{Summary}

Dimming and brightening of SSR during 1961-2005 in China is investigated in this study. One focus of this study is to present how SSR is further evolving into the new millennium. These results are inferred from daily ground-based observational records at 45 pyranometer stations. The role of TCC played in this interesting phenomenon is qualitatively studied by determining the degree of agreement between the high and low frequency parts of SSR and TCC. A statistical method is further applied to quantitatively estimate how much SSR variability is attributable to TCC frequency changes. Significant contribution by TCC changes to the dimming and brightening is revealed in this study.

There is a very complex spatio-temporal variation in decadal trends in SSR. An outstanding feature during 19611990 is dimming in nearly all seasons. Dimming is still occurring during 1991-2005 in North China, but a slight brightening appears in South China.

The inter-annual variation of SSR is dominantly determined by TCC changes, which is confirmed by a strong negative correlation between the low frequency parts of TCC and SSR.

Nearly $100 \%$ of dimming during 1961-1990 is attributable to declined atmospheric transparency, a portion of which is offset by TCC frequency changes. This feature is more pronounced in the Northeast and Southwest China regions. In the Northwest region, TCC frequency changes do contribute to the dimming in all seasons except in autumn, and the percentage attributable to this effect in each season is $16 \%, 24 \%$, and $11 \%$ in spring, summer and winter, respectively. In addition, about $10 \%$ of spring and winter dimming during 19611990 in Southeast China is likely attributable to TCC frequency changes.

Dimming of SSR during 1991-2005 in North China primarily results from TCC frequency changes. This feature is more pronounced in summer when no less than $80 \%$ of dimming is explained by TCC frequency changes. Increased atmospheric transparency generally results in an increase of SSR of 1-3\% per decade during 1991-2005 in South China, which is enhanced by TCC frequency changes in spring and autumn.

Acknowledgements. The radiation and cloud data were provided by the Climate Data Center, Chinese Meteorological Administration. This research was supported by the Knowledge Innovation Program of the Chinese Academy of Sciences (KZCX2-YW-QN201), the National Basic Research Program of China (2009CB723904) and the National Natural Science Foundation of China (40775009 and 40875084).

Topical Editor P. M. Ruti thanks one anonymous referee for her/his help in evaluating this paper.

\section{References}

Che, H., Shi, G., Zhang, Y., et al.: Analysis of 40 years of solar radiation data from China, 1961-2000, Geophys. Res. Lett., 32(6), $1-5,2005$.

Che, H., Zhang, Y., Li, Y., et al.: Horizontal visibility trends in China 1981-2005, Geophys. Res. Lett., 34, L24706, doi:10.1029/2007GL031450, 2007.

Hoyt, D.: Inter-annual cloud-cover variations in the contiguous United States, J. Appl. Meteorol., 34(11), 1824-1826, 1978.

Kaiser, D.: Analysis of total cloud amount over China, 1951-1994, Geophys. Res. Lett., 25, 3599-3602, 1998.

Karl, T. and Knight, R.: Secular trends of precipitation amount, frequency, and intensity in the United States, B. Am. Meteorol. Soc., 79(2), 231-241, 1998.

$\mathrm{Li}, \mathrm{X}$. and Zhou, X.: Analysis of the solar radiation variation of the China in recent 30 years, Quart. J. Appl. Meteor., 9, 24-31, 1998.

Li, Z., Chen, H., Cribb, M., et al.: Preface to special section on East Asian Studies of Tropospheric Aerosols: An International Regional Experiment (EAST-AIRE), J. Geophys. Res., 112(D22), 1-10, 2007a.

Li, Z., Xia, X., Cribb, M., et al.: Aerosol optical properties and their radiative effects in northern China, J. Geophys. Res., 112(D22), 1-11, 2007b.

Liang, F. and Xia, X. A.: Long-term trends in solar radiation and the associated climatic factors over China for 1961-2000, Ann. Geophys., 23, 2425-2432, 2005, http://www.ann-geophys.net/23/2425/2005/. 
Liepert, B. G.: Observed reductions of surface solar radiation at sites in the United States and worldwide from 1961 to 1990, Geophys. Res. Lett., 29, 1421, doi:10.1029/2002GL014910, 2002.

Lipert, B., Feichter, J., Lohmann, U., and Roeckner, E.: Can aerosols spin down the water cycle in a warmer and moister world?, Geophys. Res. Lett., 31(6), 1-4, 2004.

Luo Y., Lu, D., Zhou, X., et al.: Characteristics of the spatial distribution and yearly variation of aerosol optical depth over China in last 30 years, J. Geophys. Res., 106(D13), 14501-14513, 2001.

Ma, Y., Liu, X., and Xu, S.: The description of Chinese radiation data and their quality control procedures (in Chinese), Meteor. Sci., 2, 53-56, 1998.

Makowski, K., Jaeger, E., Chiacchio, M., et al.: On the relationship between diurnal temperature range and surface solar radiation in Europe, J. Geophys. Res., 114(4), 1-16, 2009.

Norris, J. R. and Wild, M.: Trends in aerosol radiative effects over China and Japan inferred from observed cloud cover, solar "dimming," and solar "brightening", J. Geophys. Res., 114, 1-11, doi:10.1029/2008JD011378, 2009.

Qian, Y., Kaiser, D., Leung, L., and Xu, M.: More frequent cloud free sky and less surface radiation in China from 1955 to 2000, Geophys. Res. Lett., 33, L01812, 1-4, 2006.

Qian, Y., Wang, W., Leung, L., Kaiser, D.: Variability of solar radiation under cloud-free skies in China: the role of aerosols, Geophys. Res. Lett., 34, L12804, 1-5, 2007.

Qiu, J. and Yang, L.: Variation characteristics of atmospheric aerosol optical depths and visibility in North China during 19801994, Atmos. Environ., 34, 603-609, 2000.

Qiu, J., Xu, X., and Yan, J.: An accuracy estimation of global solar radiation measurements at meteorological observations in China, J. Appl. Meteo. Sci., 19, 287-296, 2008.

Ren, G., Guo, J., Xu, M., et al.: Climate changes of mainland China over the past half century, Acta Meteorol. Sin., 63, 942-955, 2005.
Shi, G., Chen, Z., Wang, B., et al.: Data quality assessment and teh long-term trend of ground solar radiation in China, J. Appl. Met. Climatol., 47, 1006-1016, 2008.

Stanhill, G. and Cohen, S.: Global dimming: a review of the evidence for a widespread and significant reduction in global radiation with discussion of its probable causes and possible agricultural consequences, Agric. For. Meteor., 107, 255-278, 2001.

Wild, M., Grieser, J., and Schar, C.: Combined surface solar brightening and increasing greenhouse effect support recent intensification of the global land-based hydrological cycle, Geophys. Res. Lett., 35, L17706, 1-5, 2008.

Wild, M., Trussel, B., Ohmura, A., et al.: Global dimming and brightening: an update beyond 2000, J. Geophys. Res., 114(10), 1-14, 2009a.

Wild, M.: Global dimming and brightening: A review, J. Geophys. Res., 114, D00D16, doi:10.1029/2008JD011470, 2009b.

Xia, X., Chen, H., Wang, P., and Liang, F.: Analysis of downwelling surface solar radiation in China from National Centers for Environmental Prediction reanalysis, satellite estimates, and surface observations, J. Geophys. Res., 111(D9), 1-9, 2006.

Xia, X., Li, Z., Holben, B., Wang, P., Eck, T., Chen, H., Cribb, M., and Zhao, Y.: Aerosol optical properties and radiative effects in the Yangtze Delta region of China, J. Geophys. Res., 112, D22S12, doi:10.1029/2007JD008859, 2007.

Xia, X.: Spatio-temporal changes in sunshine duration and cloud amount as well as their relationship in China from 1954-2005, J. Geophys. Res., 115, D00K06, doi:10.1029/2009JD012879, 2010. 